\title{
CARACTERIZACIÓN DE DIFERENTES COMPOST PARA SU USO COMO COMPONENTE DE SUSTRATOS
}

\section{CHARACTERIZATION OF DIFFERENT COMPOST FOR USE AS COMPONENT OF SUBSTRATES}

\author{
Lorena Barbaro ${ }^{*}$, Monica Karlanian², Pedro Rizzo ${ }^{3}$, N Nicolas Riera ${ }^{3}$ \\ ${ }^{1}$ EEA Cerro Azul - INTA, Ruta Nacional 14. Km. 836 (3313), Cerro Azul, Misiones, Argentina. Correo \\ electrónico: barbaro.lorena@inta.gob.ar \\ ${ }^{2}$ Instituto de Floricultura - INTA, De los Reseros y Las Cabañas (1686), Hurlingham, Buenos Aires, \\ Argentina. Correo electrónico: karlanian.monica@inta.gob.ar \\ ${ }^{3}$ Instituto de Microbiología y Zoología Agrícola - INTA, De los Reseros y Las Cabañas (1686), \\ Hurlingham. Buenos Aires, Argentina. Correo electrónico: rizzo.pedro@inta.gob.ar; riera.nicolas@inta. \\ gob.ar \\ *Autor para correspondencia E-mail: barbaro.lorena@inta.gob.ar
}

\section{RESUMEN}

Actualmente el uso de sustrato se ha incrementado en varios sectores agrícolas. Entre los materiales empleados para su formulación se encuentran los compost, y como todo componente es importante conocer sus propiedades previo a su uso. El objetivo de este trabajo fue caracterizar 15 compost de diferentes orígenes, para evaluarlos como componente de sustrato. Se analizaron los siguientes parámetros: densidad aparente, espacio poroso total, capacidad de retención de agua (CRA), porosidad de aireación (PA), granulometría, $\mathrm{pH}$, conductividad eléctrica $(\mathrm{CE})$, concentración de calcio, magnesio, potasio, sodio y nitratos. Hubo diferencias significativas $(P<0,05)$ entre los compost para todas las variables analizadas. La densidad aparente fue mayor en el compost de ave de corral. El compost de corteza de pino gruesa tuvo el mayor porcentaje de PA y el compost de residuos de poda el mayor porcentaje de CRA. Los compost de corteza de pino podrían ser componentes que aportarían aireación en una formulación de sustrato. Los valores de $\mathrm{pH}$ obtenidos se encontraron entre 4,7 a 8,7 y de CE entre 0,09 a 5,55 dS m $\mathrm{m}^{-1}$. La mayoría de los compost superaron el rango adecuado de $\mathrm{pH}(5,5-6,3)$ y si bien la mayoría no tuvieron una CE elevada, hubo algunos como el compost de ave de corral, de residuos sólidos urbanos y de residuos porcinos que superaron $1 \mathrm{dS} \mathrm{m}^{-1}$, y podrían causar efectos nocivos en las plantas. Todos los compost son viables para su uso teniendo en cuenta sus propiedades y las condiciones del cultivo en que se va a utilizar el sustrato formulado.

Palabras clave: cultivo sin suelo, recipiente, compost, aves, cerdos, residuos urbanos, corteza pino, orujo uva, caña azúcar.

\section{ABSTRACT}

The use of substrates in agriculture has increased in recent years. Compost is one of the materials used in substrate formulation, and therefore, it is important to know its properties prior to its use. The objective of this study was to characterize 15 different types of compost from different sources in order to evaluate them as substrate components. The following parameters were determined:

Recibido: 30 octubre 2018. Aceptado: 14 enero 2019. 
bulk density, total pore space, water retention capacity (CRA), aeration porosity (PA), granulometry, $\mathrm{pH}$, electrical conductivity (EC), and concentrations of calcium, magnesium, potassium, sodium and nitrates. There were significant differences $(P<0.0001)$ among the compost for all the variables analyzed. Poultry compost had the highest bulk density. Thick pine bark compost had the highest percentage of PA, while pruning waste compost recorded the highest percentage of CRA. Pine bark compost could increase aeration in a substrate formulation. The $\mathrm{pH}$ values obtained ranged from 4.7 to 8.7, while CE values varied between 0.09 and $5.55 \mathrm{dS} \mathrm{m}^{-1}$. Most of the compost exceeded an optimal $\mathrm{pH}$ range (5.5-6.3). On the contrary, most compost did not reach high EC values. However, some types of compost, such as those from poultry, urban solid waste and pig waste, exceeded $1 \mathrm{dS} \mathrm{m}^{-1}$, and could cause harmful effects on plants. All types of compost are viable for use provided both compost properties and crop conditions are considered to decide which substrate to use.

Key words: soilless culture, container, compost, poultry, pigs, city residues, pine cortex, grape byproduct, sugarcane.

\section{INTRODUCCIÓN}

Actualmente el uso de sustrato se ha incrementado, y se debe especialmente a que se está sustituyendo cada vez más el cultivo tradicional en suelo por el cultivo hidropónico y en sustrato (Urrestarazu, 2015). Esta sustitución deriva de distintas razones, entre ellas, algunos factores que limitan la continuidad de cultivos intensivos en el suelo como los agentes fitopatógenos, el incremento de la salinidad y la degradación del suelo (Putra y Yuliando, 2015; Palencia et al., 2016). Como también, la necesidad de transportar plantas completas a distintos lugares de donde fueron cultivadas (Raviv y Lieth, 2008). Por lo tanto, el sustrato está siendo usado en varios sectores agrícolas, si bien es más pronunciado el uso en el sector hortícola (Kleiber et al., 2012) y ornamental (Bunt, 2012), existen otros sectores como el forestal (Landis y Morgan, 2009; Aguilera Rodríguez et al., 2016; Gonzaga et al., 2016), frutícola (Abbas et al., 2015; Palencia et al., 2016) y diversos cultivos industriales (Biaggi et al., 2011; Krieger et al., 2010) que se emplea principalmente para la producción de plantines.

Un sustrato es cualquier material sólido y poroso diferente del suelo in situ que se encuentra dentro de un recipiente, sirve de anclaje a las raíces de la planta y permite una libre circulación del agua y de los gases (Carmona y Abad, 2008). Para que estas funciones se cumplan se deben utilizar para su formulación materiales adecuados. Estos materiales serán seleccionados teniendo en cuenta una serie de propiedades físicas y químicas que están condicionadas por el sistema de cultivo, es decir, si se cultiva fuera o bajo invernáculo; la altura, volumen y forma del recipiente; tipo de sistema de riego y características del agua de riego. Como también, los requerimientos de la especie, la época del año y duración del ciclo o parte del ciclo en que la planta desarrollara en el sustrato (Abad et al., 2004; Vence, 2008).

Entre las propiedades físicas necesarias se encuentra la densidad aparente, la cual debería ser baja, para facilitar las actividades durante el manejo del cultivo (Bunt, 2012). La distribución de partículas, propiedad de la que dependerá la distribución del tamaño de los poros y, como consecuencia, el porcentaje de poros con aire y agua que contendrá el sustrato (Carlile, 2015). Además, una correcta aireación aportará oxígeno al sistema radicular y permitirá la evacuación del gas carbónico producido por las raíces y microorganismos (Morales Maldonado y Casanova Lugo, 2015). Y una correcta capacidad de retención de agua permitirá que la planta tenga agua disponible (Gayosso-Rodríguez et al., 2016). Entre las principales propiedades químicas responsables de la calidad del sustrato se mencionan el $\mathrm{pH}$ y la conductividad eléctrica (Sonneveld y Voogt, 2009; Barbaro et al., 2018). Es recomendable que el sustrato tenga un $\mathrm{pH}$ ligeramente ácido, para que los nutrientes se encuentren disponibles, y una baja conductividad eléctrica, para que no existan problemas de toxicidad por sales.

Raras veces un material o componente tiene todas las condiciones físicas y químicas adecuadas para el sistema de cultivo requerido, es por eso que en la mayoría de los casos es necesario mezclarlo con otros materiales (Abad et al., 2004; Normann et al., 2006;). Sin embargo, se recomienda evitar la incorporación de un número elevado de componentes (Normann et al., 2006). Por lo cual es muy importante conocer las propiedades de cada material para evitar pérdidas de tiempo e incurrir en gastos innecesarios (Carlile et al., 2015). Por tal motivo, se aconseja realizar los análisis físicos y químicos con metodologías específicas para sustratos tanto a cada material como al sustrato formulado.

Son varios los materiales que se emplean para la formulación de sustratos; entre los inorgánicos se destaca la perlita expandida, vermiculita exfoliada, lana roca, cenizas volcánicas y lecas, y entre los orgánicos, las turbas y residuos y/o 
subproductos de diversas actividades industriales o explotaciones (Bunt, 2012; Cruz et al., 2013). Estos últimos en su mayoría deben pasar por algún proceso de estabilización de la materia orgánica, siendo el más empleado el compostaje (Cáceres et al., 2015).

Los compost actualmente son ampliamente utilizados como componentes en la formulación de los sustratos (Barrett et al., 2016), pero como todo material también es importante conocer sus propiedades antes de emplearlo. De acuerdo a estas propiedades un compost se utilizará en forma pura o en mezclas entre $20 \%$ a $80 \%$ con otros componentes (Carmona y Abad, 2008).

En base a lo mencionado, en este trabajo se caracterizaron mediante análisis físicos y químicos 15 compost de diferentes orígenes para evaluar su potencial como componente de sustrato.

\section{MATERIALES Y METODOS}

\section{Compost evaluados}

Los compost evaluados fueron obtenidos en diferentes empresas argentinas dedicadas a elaborar este producto con fines comerciales, y en establecimientos del INTA (Instituto Nacional de Tecnología Agropecuaria). En la Tabla 1 se presenta el tipo de residuo o conjunto de residuos que se utilizó para elaborar cada compost evaluado.

Compost de ave de corral (CAC (1) y (2)): El residuo avícola utilizado para elaborar los compost fue la cama o lecho de gallinas ponedoras criadas en galpones automatizados con instalaciones tipo Zucami ubicados en la localidad de Mercedes (Buenos Aires, Argentina). Ambos fueron elaborados en el Instituto de Microbiología y Zoología Agrícola (IMYZA) del INTA durante un período de 83 días. El sistema de compostaje utilizado fue mediante pilas con volteo; se realizaban controles de temperatura y se mantuvo la humedad con riegos periódicos.

Compost de residuos porcino (CRP): Este compost se elaboró con la cama o lecho de los porcinos producidos mediante un sistema de lecho profundo. Este sistema consiste en recriar y terminar cerdos en grupos numerosos en un mismo compartimento, con comederos y bebederos automáticos y adición constante de grandes volúmenes de cama o lecho (paja de cereales, virutas de madera, etc.). En este caso, el residuo utilizado como lecho fue la paja de trigo. El compost fue elaborado en la Estación

Tabla 1. Compost evaluados, abreviatura y residuo utilizados para la elaboración de cada compost. Table 1. Types of compost, abbreviation and waste used for the elaboration of each compost.

\begin{tabular}{|c|c|c|}
\hline Compost evaluados & Abreviatura & Residuo utilizado para su elaboración \\
\hline \multirow[t]{4}{*}{ Compost de ave de corral (1) } & CAC (1) & $40 \%$ de guano de gallinas ponedoras \\
\hline & & $20 \%$ de mazorca de maíz triturada \\
\hline & & $20 \%$ de aserrín de pino \\
\hline & & $20 \%$ de viruta de pino \\
\hline \multirow[t]{3}{*}{ Compost de ave de corral (2) } & CAC (2) & $60 \%$ de guano de gallinas ponedoras \\
\hline & & $20 \%$ de mazorca de maíz triturada \\
\hline & & $20 \%$ de aserrín de pino \\
\hline \multirow[t]{2}{*}{ Compost porcino } & CRP & $50 \%$ orina y excrementos \\
\hline & & $50 \%$ de paja de trigo \\
\hline Compost RSU (1) & CRSU (1) & Residuos sólidos urbano sin clasificar \\
\hline Compost RSU (2) & CRSU (2) & Fracción orgánica de los residuos sólidos urbanos \\
\hline Compost corteza de pino fina & $\mathrm{CCPF}$ & Corteza de pino \\
\hline Compost corteza de pino gruesa & CCPG & Corteza de pino \\
\hline Compost corteza de pino media (1) & ССРM (1) & Corteza de pino \\
\hline Compost corteza de pino media (2) & ССРM (2) & Corteza de pino \\
\hline Compost de poda de mora & $\mathrm{CPM}$ & Restos de poda de árboles de Morus alba L. \\
\hline Compost de poda de cedro & СРC & Restos de poda de árboles de Cedrus spp. \\
\hline Compost de de poda de fresno & $\mathrm{CPF}$ & Restos de poda de árboles de Fraxinus americana $\mathrm{L}$. \\
\hline Compost de poda & $\mathrm{CP}$ & Restos de poda del arbolado urbano \\
\hline Compost de orujo de uva agotado & COUA & Orujo de uva agotado \\
\hline Compost de bagazo de caña azúcar & CBCA & Bagazo de caña de azúcar \\
\hline
\end{tabular}


Experimental Agropecuaria (EEA) Marcos Juárez del INTA en la provincia de Córdoba (Argentina) durante un periodo de 85 días. El sistema de compostaje utilizado fue mediante hileras con volteo mediante tractores con palas mecánicas, se realizaron controles de temperatura y se mantuvo la humedad con riegos periódicos.

Compost de residuos sólidos urbanos (CRSU (1) y (2)): El compost de RSU 1 fue elaborado en la planta de residuos domiciliarios ubicada en CEAMSE (Coordinación Ecológica Área Metropolitana Sociedad del Estado) la cual es una empresa pública argentina encargada de la gestión de RSU del Gran Buenos Aires, incluyendo la Ciudad de Buenos Aires. Esta empresa utilizó los residuos sin clasificar para elaborar el compost. El sistema de compostaje utilizado fue mediante hileras estáticas con aireación forzada.

El compost de RSU 2 fue elaborado en la localidad de Trenque Lauquen (Buenos Aires, Argentina) con los RSU de la ciudad. En este caso, para elaborar el compost se clasificaron los residuos y se seleccionó la parte orgánica como materia prima para la elaboración del compost. El sistema de compostaje utilizado fue mediante pilas con volteo.

Compost corteza de pino fina y gruesa (CCPF y CCPG): Ambos compost fueron adquiridos en la empresa Terrafertil S.A., ubicada en la localidad de Moreno (Buenos Aires, Argentina). Para producir los compost se emplearon como materia prima corteza de pino (Pinus elliotti Engelm. y Pinus taeda L.) proveniente de distintos aserraderos. El sistema de compostaje utilizado fue mediante hileras con volteo, con riegos periódicos y control de la relación de nitrato y amonio. A las hileras se incorporó urea en forma líquida y los volteos eran semanales mediante un tractor con pala mecánica. Luego del proceso de compostaje el compost obtenido se pasó por una malla de $14 \mathrm{~mm}$ y otra de $8 \mathrm{~mm}$ separando por un lado al compost fino $(<8 \mathrm{~mm})$ y por otro, el compost grueso (8 a $14 \mathrm{~mm}$ ).

Compost corteza de pino media (CCPM (1) y (2)): Estos compost fueron elaborados a partir de corteza de pino (Pinus elliotti Engelm. y Pinus taeda L.) de distintos aserraderos, el CCPM 1 se elaboró en una empresa ubicada en la localidad de Virasoro (Corrientes, Argentina) y el CCPM 2 se realizó en la empresa Don Cesareo S.C.A ubicada en la localidad de Candelaria (Misiones, Argentina). Para elaborar ambos compost se utilizó el sistema de hileras con volteo mediante tractor con pala mecánica, se realizaron riegos periódicos y se incorporó urea granulada.
La duración del compostaje fue de 6 meses aproximadamente.

Compost de poda (CP), de mora (CPM), cedro (CPC) y fresno (CPF): Los compost fueron elaborados en las instalaciones del Vivero Municipal del Instituto Municipal de Desarrollo Económico Local (IMDEL) de la localidad de Moreno (Buenos Aires, Argentina). Se utilizó el sistema de hileras con volteo manual mediante palas, se realizaban controles de temperatura y se mantuvo la humedad con riegos periódicos. La materia prima empleada fue el resto de poda perteneciente al arbolado urbano de la localidad de Moreno. A medida que se podaron los arboles los restos de poda fueron triturados mediante una máquina "chipeadora" para luego llevar el material obtenido al Vivero Municipal y realizar el proceso de compostaje. Este proceso duró aproximadamente 6 meses. El compost de poda (CP) fue obtenido a partir de la mezcla de todos los restos de poda, en cambio, el compost de mora (Morus alba L.), cedro (Cedrus spp.) y fresno (Fraxinus americana L.) fueron separados desde su recolección para compostarlos por separado y así obtener un compost del residuo de cada especie.

Compost de orujo de uva agotado (COUA): La elaboración del compost de orujo de uva agotado se realizó en la EEA Mendoza del INTA. La materia prima utilizada fue el orujo de uva agotado, residuo obtenido luego de destilar el orujo de uva para la obtención de etanol. Este residuo se extrajo de la destilaría Derivados Vínicos S.A., ubicada en el departamento de San Martín, Mendoza (Argentina). El proceso de compostaje fue mediante el sistema de pilas con volteo, se controló la temperatura y mantuvo la humedad mediante riegos periódicos. El proceso duró aproximadamente 5 meses.

Compost de bagazo de caña azúcar (CBCA): Este compost fue elaborado en la EEA Faimallá del INTA en la provincia de Tucumán (Argentina), y la materia prima empleada fue el residuo de los tallos de la caña de azúcar (Saccharum officinarum) después de pasar por el proceso de extracción del jugo azucarado en el ingenio azucarero. A este residuo se lo llama bagazo de caña de azúcar. El sistema de compostaje utilizado fue mediante pilas con volteo.

\section{Análisis físicos y químicos}

Se analizaron las muestras de cada compost de la siguiente manera: se pasó todo el material (80 litros) a través de un tamiz con una malla de 20 $\mathrm{mm} \times 20 \mathrm{~mm}$. Luego se mezcló y se trazaron dos líneas oblicuas a cada lote tomando 6 litros de dos cuartos opuestos, conformando una submuestra 
de 12 litros. En cada submuestra se ajustó el porcentaje de humedad (\% peso) a un $50 \%$ ( \pm 2) y se analizaron las siguientes variables por triplicado en el Laboratorio de Sustratos y Aguas del Instituto de Floricultura del INTA (34²36' S; $58^{\circ} 40^{\prime} \mathrm{O}$ ). Las variables analizadas fueron:

Densidad aparente: se determinó mediante el método de Hofmann (Fermino, 2003).

Espacio poroso total, capacidad de retención de agua y porosidad de aire: se empleó el método de De Boodt mediante los lechos de arena (De Boodt et al., 1974).

Granulometría: se determinó mediante una tamizadora vibratoria con tamices de 5,56; 4,75; 3,$35 ; 2,0 ; 1,4 ; 1$ y $0,5 \mathrm{~mm}$, durante 10 minutos en forma intermitente (Ansorena Miner, 1994). Los resultados se expresaron en porcentaje de tamaño de partículas $>3,35 \mathrm{~mm}$, entre 3,35 a $1 \mathrm{~mm} \mathrm{y}<$ $1 \mathrm{~mm}$.

Conductividad eléctrica (CE) y pH: se analizó en una relación $1+5 \mathrm{vol} / \mathrm{vol}$ de compost/agua, en un frasco de $250 \mathrm{~mL}$ se colocaron $150 \mathrm{~mL}$ de agua destilada y se llevó a $180 \mathrm{~mL}$ con el agregado de sustrato. Se agitó durante 10 minutos y se dejó reposar 15 minutos, luego se realizaron las mediciones en la solución con un conductímetro (Thermo Orion modelo 145) y un peachímetro (Horiba M-12) (Barbaro et al., 2011).

Concentración de calcio, magnesio, potasio y sodio: fueron analizados en el filtrado de la solución $1+5 \mathrm{vol} / \mathrm{vol}$ descrita en el párrafo anterior, con un espectrofotómetro de absorción atómica (Varian modelo 220 A, Mulgrave Victoria, Australia) y los nitratos con electrodo ión selectivo (WTW modelo Inolab 7320, Weiheim, Alemania). Los resultados se expresaron en $\mathrm{mg}$ $\mathrm{L}^{-1}$ de compost.

\section{Análisis estadísticos}

Con los datos obtenidos de los análisis físicos y químicos realizados a cada compost se realizó un análisis de varianza y Test de Tukey $(\mathrm{P}<0,05)$ para comparación de medias. Para el análisis de correlación del $\mathrm{pH}$ y la $\mathrm{CE}$ con los nutrientes se utilizó el coeficiente de correlación de Pearson. El software estadístico utilizado fue el programa InfoStat versión 2009 (Di Rienzo et al., 2009).

\section{RESULTADOS Y DISCUSION}

\section{Propiedades químicas}

Los problemas nutritivos más comunes ocurren en los cultivos cuando el $\mathrm{pH}$ se encuentra fuera del rango óptimo (Ansorena Miner, 1994; Bures, 1997; Barbaro et al., 2018). Además, la gran mayoría de las especies cultivadas en sustratos se desarrollan en rangos de $\mathrm{pH}$ entre 5,5 y 6,3 (Abad et al., 2001). En base a lo expuesto, los valores de $\mathrm{pH}$ obtenidos (Tabla 2) se encontraron en un rango de 4,7 a 8,7. El CAC (1) tuvo el mayor valor de $\mathrm{pH}$ y se diferenció significativamente de los otros compost $(\mathrm{P}<0,05)$, seguido por el CPM y CRP entre los cuales no hubo diferencia. Entre el CRSU (2), el CAC (2), el COUA, el CPF, el CRSU (1) y el CP hubo diferencias $(P<0,05)$ y se encontraron por encima del rango mencionado. Los valores de $\mathrm{pH}$ más bajos y menores al rango adecuado, fueron en el CCPM (2), en el CBCA, en el CCPG y en el CCPF con diferencia entre sí $(\mathrm{P}<0,05)$. El CCPF, el CCPG, el CCPM (1) y el $\mathrm{CPC}$ fueron los únicos que se hallaron dentro del rango óptimo.

En general mientras el proceso de compostaje avanza el valor de $\mathrm{pH}$ aumenta hasta valores entre 6,5 a 8 dependiendo del material (Carmona y Abad, 2008), consecuencia por lo cual el $60 \%$ de los compost evaluados superaron el valor de 6,3. Según Carrión et al. (2005) los compost son alcalinos debido a que contienen menor proporción de iones intercambiables dehidrógeno y mayor de calcio y magnesio, y en algunos casos también de sodio. En coincidencia, los compost que resultaron con mayores valores de $\mathrm{pH}$ contenían una alta concentración de sodio (CRSU (1), CRP y CAC (1)) o una alta concentración de calcio y magnesio (CP y CPM). Asimismo, las concentraciones de calcio, magnesio y sodio tuvieron correlaciones de 0,48 a 0,69 en relación al pH (Tabla 3).

Existen técnicas para corregir el $\mathrm{pH}$ del componente o sustrato formulado, si el valor de $\mathrm{pH}$ es inferior al rango, se recomienda corregir el material con cal, caliza, dolomita o mezclando con otros materiales ácidos, y en el caso de superar el rango, mediante la adición de azufre, sulfato ferroso, sulfato de aluminio $\mathrm{u}$ otros compuestos azufrados (Abad et al., 1993; Barbaro et al., 2010).

Con respecto a la $\mathrm{CE}$, valores bajos en un sustrato permitiría que se puedan manejar las concentraciones de nutrientes minerales según los requerimientos del cultivo mediante la fertilización (Landis et al., 2000). Valores mayores a $1 \mathrm{dSm}^{-1}(1+5 \mathrm{v} / \mathrm{v})$, límite establecido para la metodología empleada (Barbaro et al., 2014) podrían disminuir el desarrollo de las plantas debido a desbalances nutricionales y/o efectos fitotóxicos. En relación a los compost evaluados los valores de CE obtenidos (Tabla 1) se hallaron entre $0,09 \mathrm{dSm}^{-1}$ y $5,55 \mathrm{dSm}^{-1}$. El CRP tuvo el mayor valor diferenciándose $(\mathrm{P}<0,05)$ con todos los compost, seguido por el CRSU (1) y el CAC (1). Estos compost superaron ampliamente el límite mencionado, por lo cual si se los emplean en forma pura como sustrato provocarían efectos nocivos en la planta. 
Tabla 2. Valores de $\mathrm{pH}$, conductividad eléctrica (CE), concentración soluble de nitrato, calcio, magnesio, potasio y sodio de las muestras de compost analizadas.

Table 2. Values of $\mathrm{pH}$, electrical conductivity (EC), soluble concentration of nitrate, calcium, magnesium, potassium and sodium of the compost analyzed.

\begin{tabular}{|c|c|c|c|c|c|c|c|}
\hline $\begin{array}{l}\text { Muestras } \\
\text { de } \\
\text { compost }\end{array}$ & $\mathrm{pH}$ & $\begin{array}{c}C E \\
\mathrm{~d} S \mathrm{~m}^{-1}\end{array}$ & Nitratos & Calcio & Magnesio & Potasio & Sodio \\
\hline & & & & 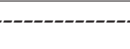 & $\mathrm{g} \mathrm{L}^{-1}$ de com & st ------ & ------- \\
\hline CAC (1) & 8,72 a & $2,66 \mathrm{c}$ & $223 \mathrm{j}$ & 2216 c & $466 \mathrm{f}$ & $11354 \mathrm{~b}$ & $2884 \mathrm{c}$ \\
\hline CAC (2) & $7,02 \mathrm{~g}$ & $1,12 \mathrm{~d}$ & $2917 \mathrm{~b}$ & $534 \mathrm{~h}$ & $1067 \mathrm{~b}$ & $1883 \mathrm{i}$ & $462 \mathrm{~h}$ \\
\hline CRP & $8,30 \mathrm{~b}$ & 5,55 a & $2923 \mathrm{~b}$ & $116 \mathrm{i}$ & $404 \mathrm{~h}$ & 15949 a & $11465 \mathrm{~b}$ \\
\hline CRSU (1) & $7,89 \mathrm{~d}$ & $5,24 \mathrm{~b}$ & 421 & 3103 a & 1174 a & $5255 \mathrm{~g}$ & 11718 a \\
\hline CRSU (2) & $6,76 \mathrm{~h}$ & $1,13 \mathrm{~d}$ & 361 & $872 \mathrm{f}$ & 767 e & $2633 \mathrm{~h}$ & $1267 \mathrm{~d}$ \\
\hline $\mathrm{CCPF}$ & $5,42 \mathrm{k}$ & 0,091 & 1973 c & 4 o & $6 \mathrm{~lm}$ & 195 o & 1381 \\
\hline CCPG & 5,261 & $0,17 \mathrm{k}$ & $24 \mathrm{~m}$ & 141 & $18 \mathrm{k}$ & $221 \mathrm{n}$ & $128 \mathrm{k}$ \\
\hline ССРМ (1) & $5,75 \mathrm{j}$ & $0,30 \mathrm{ij}$ & $480 \mathrm{i}$ & $7 \mathrm{~lm}$ & 161 & 2851 & $88 \mathrm{~m}$ \\
\hline ССРМ (2) & $4,72 \mathrm{n}$ & $0,42 \mathrm{~h}$ & 3454 a & $7 \mathrm{mn}$ & $5 \mathrm{~m}$ & $213 \mathrm{~m}$ & $67 n$ \\
\hline CPM & $8,39 \mathrm{~b}$ & $0,74 \mathrm{f}$ & $1827 \mathrm{~d}$ & $1675 \mathrm{~d}$ & 852 d & $5931 \mathrm{f}$ & $584 \mathrm{e}$ \\
\hline СРC & $6,22 \mathrm{i}$ & $0,35 \mathrm{ij}$ & 1757 e & $795 \mathrm{~g}$ & $263 \mathrm{i}$ & $6381 \mathrm{e}$ & $516 \mathrm{f}$ \\
\hline $\mathrm{CPF}$ & $7,57 \mathrm{e}$ & $0,53 \mathrm{~h}$ & $1449 \mathrm{~g}$ & $983 \mathrm{e}$ & $436 \mathrm{~g}$ & 10603 c & $474 \mathrm{~g}$ \\
\hline $\mathrm{CP}$ & $8,12 \mathrm{c}$ & $1,05 \mathrm{f}$ & $1583 \mathrm{f}$ & $2862 \mathrm{~b}$ & $887 \mathrm{c}$ & $6543 \mathrm{~d}$ & $371 \mathrm{i}$ \\
\hline COUA & $7,29 \mathrm{f}$ & $0,39 \mathrm{i}$ & $522 \mathrm{~h}$ & $42 \mathrm{j}$ & 151 & 1692 j & $265 \mathrm{j}$ \\
\hline CBCA & $5,05 \mathrm{~m}$ & $0,33 \mathrm{jk}$ & $56 \mathrm{k}$ & $22 \mathrm{k}$ & $33 \mathrm{j}$ & $705 \mathrm{k}$ & 831 \\
\hline
\end{tabular}

* Compost de ave de corral (CAC (1) y (2)), compost de residuos porcino (CRP), compost de residuos sólidos urbanos (CRSU (1) y (2)), compost corteza de pino fina (CCPF), compost corteza de pino gruesa (CCPG), compost corteza de pino media (CCPM (1) y (2)), compost de poda de mora (CPM), compost de poda de cedro (CPC), compost de poda de fresno $(\mathrm{CPF})$, compost de poda (CP), compost de orujo de uva agotado (COUA) y compost de bagazo de caña azúcar (CBCA).

** Letras distintas entre filas de una misma columna indican diferencias significativas $(p \leq 0,05)$ para el test de Tukey.

* Poultry compost (CAC (1) and (2)), pig waste compost (CRP), urban solid waste compost (CRSU (1) and (2)), fine pine bark compost (CCPF), thick pine bark compost (CCPG), medium pine bark compost (CCPM (1) and (2)), mulberry prune compost $(\mathrm{CPM})$, cedar pruning compost $(\mathrm{CPC})$, ash pruning compost $(\mathrm{CPF})$, pruning compost $(\mathrm{CP})$, grape marc compost (COUA) and sugarcane bagasse compost (CBCA).

** Different letters between rows of the same column indicate significant differences $(p \leq 0.05)$ for the Tukey test.

Tabla 3. Matriz de coeficientes de correlación de Pearson, entre las variables químicas analizadas a los compost.

Table 3. Matrix of Pearson correlation coefficients for the chemical variables analyzed to the compost

\begin{tabular}{|c|c|c|c|c|c|c|c|c|}
\hline \multirow{3}{*}{ Variables } & & & & & & & & \\
\hline & & \multirow[t]{2}{*}{$\mathrm{pH}$} & \multirow{2}{*}{$\begin{array}{c}\mathrm{CE} \\
\mathrm{dS} \mathbf{m}^{-1} \\
\end{array}$} & \multirow[t]{2}{*}{ Nitratos } & \multirow[t]{2}{*}{ Calcio } & Magnesio & Potasio & \multirow[t]{2}{*}{ Sodio } \\
\hline & & & & & & \multicolumn{2}{|c|}{$\mathrm{mg} \mathrm{L}^{-1}$ de compost } & \\
\hline $\mathrm{pH}$ & & 1 & & & & & & \\
\hline $\mathrm{CE}\left(\mathrm{dS} \mathrm{\textrm {m } ^ { - 1 } )}\right.$ & & 0,59 & 1 & & & & & \\
\hline Nitratos & $\vec{b}$ & $-0,01$ & 0,03 & 1 & & & & \\
\hline Calcio & 言 & 0,69 & 0,44 & $-0,22$ & 1 & & & \\
\hline Magnesio & 8 & 0,67 & 0,48 & 0,05 & 0,76 & 1 & & \\
\hline Potasio & $\vec{\sigma}$ & 0,77 & 0,65 & 0,19 & 0,39 & 0,32 & 1 & \\
\hline Sodio & 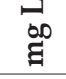 & 0,48 & 0,98 & 0,01 & 0,36 & 0,40 & 0,57 & 1 \\
\hline
\end{tabular}


Por otro lado, las concentraciones de sodio, potasio y calcio tuvieron correlaciones de 0,44 a 0,98 en relación a la CE (Tabla 3). En este sentido, el CRP tuvo la mayor concentración de potasio y el CRSU (1) tuvo la mayor concentración de calcio, magnesio y sodio diferenciándose $(\mathrm{P}$ $<0,05$ ) de los restantes compost (Tabla 2). En cuanto a los nitratos (Tabla 2), el CCPM (2) se destacó por tener la mayor concentración de este anión diferenciándose significativamente $(\mathrm{P}<0,05)$ de los demás compost, seguido por el CCA (2) y el CRP sin diferencias entre ambos. De manera contraria, en general los compost de corteza de pino tuvieron menor concentración de las variables mencionadas, resultando el CCPG con la menor concentración de nitrato, el CCPF con la menor concentración de calcio y potasio, y el CCPM (2) con la menor concentración de magnesio y sodio, con diferencias significativas $(\mathrm{P}<0,05)$. En coincidencia, los valores más bajos de CE se encontraron en el CCPG y en el CCPF con diferencia entre ambos $(\mathrm{P}<0,05)$.

En relación a lo expuesto, Barbaro et al. (2013) realizaron un ensayo en donde se evaluaron tres CAC cuya CE fue de 1,$4 ; 2,0$ y $2,7 \mathrm{dS} \mathrm{m}^{-1}$ $(1+5 \mathrm{v} / \mathrm{v})$, cada uno mezclado con $80 \%$ o $50 \%$ de compost de corteza de pino para producir plantines de coral (Salvia splendens L.) y de alegría de hogar (Impatiens walleriana hybrids Hook. f.), los sustratos formulados con $20 \%$ de cada CAC fueron en donde se obtuvo el mejor desarrollo de ambas especies de plantines. Además, se observó que a mayor porcentaje de CAC incorporado en el sustrato en el análisis foliar había una mayor concentración de potasio y menor de calcio y magnesio, es decir, que hubieron desbalances nutricionales que afectaron el desarrollo de los plantines por causa de la alta concentración de potasio, variable correlacionada con la alta CE.

También Garcia et al. (2002) concluyeron que la variable $C E$ es el factor principal que puede limitar el uso del compost en la formulación de sustratos. Estos autores evaluaron dos compost preparados a partir de residuos agroindustriales, uno con una CE de 4,26 dS m ${ }^{-1}$ y el otro de 3,7 dS $\mathrm{m}^{-1}$; en ambos se destacó una alta concentración de potasio, mezclados con un sustrato comercial en diferentes proporciones. En los sustratos con hasta $50 \%$ de compost desarrollaron mejor las plantas de Calendula officinalis y hasta un 25\% de compost desarrollaron mejor las plantas de Calceolaria pinnata L. Con este resultado también se puede destacar la importancia de conocer la tolerancia a las concentraciones de sales de la especie que se va a cultivar, ya que existen especies que toleran CE mayores al límite establecido. Por ejemplo, se han reportado un buen crecimiento de varias especies leñosas en medios que contienen compost con altos valores de CE, como Eucaliptus (Sallesses et al., 2015); Pinus pinea y Cupressus arizonica (Guerrero et al., 2002); Deutzia gracilis, Cornus alba L. Forsythia $\times$ intermedia, Physocarpus opulifolius L., Potentilla fruticosa L., Ligustrum vulgare L. y Rosa L. (Chong et al., 1991).

\section{Propiedades físicas}

Para que el manipuleo del sustrato y sus componentes sea menos dificultosa, con respecto a las labores de mezclado del sustrato, cargado en los contendedores y traslado, se recomienda que los mismos tengan una densidad aparente inferior a $400 \mathrm{~kg} \mathrm{~m}^{-3}$ (Abad et al., 2001). Además, valores mayores, incrementan los costos de transporte (Cruz, 2013). En este sentido, el CRSU (1), CAC (1) y el CRSU (2) tuvieron densidades mayores a la adecuada (Tabla 4), con diferencias significativas entre ellos $(\mathrm{P}<0,05)$ y con los demás compost, por lo cual, al momento de formular un sustrato con estos compost se los debería alivianar con otro/s componente/s. Los compost menos densos fueron el CBCA, CCPF y CCPG sin diferencias entre ellos.

El porcentaje de espacio poroso total (Tabla 4) fue mayor en el CBCA, el CPF, el CPC, el CPM, el CCPM (2), el CCPG y el CCPF, sin diferencias entre ambos, pero solo los tres primeros se diferenciaron de los restantes compost. El CAC (1) fue el de menor porcentaje diferenciándose de los otros compost. La porosidad total de un sustrato tendría que ser de al menos 85\% (Abad et al., 2001) y los compost evaluados, excepto por el CCA (1), el CRSU (1) y el CRSU (2), se encontraron muy cerca de este límite o lo superaron.

Pero más importante aún es conocer como la porosidad total está repartida entre aquel espacio ocupado por agua y aire. Por lo cual, se observó que el CCPG tuvo el mayor porcentaje de porosidad de aireación (Tabla 4 ) y se diferenció de todos los compost evaluados $(\mathrm{P}<0,05)$, seguido por el CCPF y el CBCA sin diferencias entre ambos, y el COUA y el CCPM (2) con diferencias entre ambos. Los compost CCPG, CCPF, CBCA, CCPM (2) y COUA tuvieron una alta porosidad de aireación, mayor al 40\%, considerando que los valores óptimos de porcentaje de poros con aire son entre 20 a 30\% (Abad et al., 2001). Es decir, que estos compost en un sustrato podrían incrementar el volumen de espacios con aire. En cambio, el CRSU (2), CAC (1), CRP y CP resultaron tener los menores porcentajes de porosidad de aireación.

Con respecto al porcentaje de capacidad de retención de agua (Tabla 4), fue mayor en el $\mathrm{CP}$, el CRP y el CPM, sin diferencias entre sí, seguidos por el CPF el cual se diferenció del CP y del CRP pero no del CPM $(\mathrm{P}<0,05)$. Solo estos cuatro compost se encontraron dentro del rango 
Tabla 4. Valores de densidad aparente (Dap), porosidad de aireación (PA), capacidad de retención de agua (CRA), espacio poroso total (EPT), porcentaje de tamaño de partículas $>3,35 \mathrm{~mm}$, entre 3,35 a $1 \mathrm{~mm}$ y $<1 \mathrm{~mm}$ de las muestras de compost analizadas.

Table 4. Values of bulk density (Dap.), aeration porosity (PA), water retention capacity (CRA), total porous space (EPT), percentage of particle size $>3.35 \mathrm{~mm}$, from 3.35 to $1 \mathrm{~mm}$, and $<1 \mathrm{~mm}$ of the compost analyzed.

\begin{tabular}{|c|c|c|c|c|c|c|c|}
\hline \multirow{2}{*}{$\begin{array}{l}\text { Muestras } \\
\text { de } \\
\text { compost }\end{array}$} & \multirow{2}{*}{$\begin{array}{c}\text { Dap } \\
\mathrm{kg} \mathrm{m}^{-3}\end{array}$} & \multirow[t]{2}{*}{ PA (\%) } & \multirow[t]{2}{*}{ CRA (\%) } & \multirow[t]{2}{*}{ EPT (\%) } & \multicolumn{3}{|c|}{ Tamaño de partículas (\%) } \\
\hline & & & & & $\begin{array}{c}>3,35 \\
\mathrm{~mm}\end{array}$ & $\begin{array}{r}3,35- \\
1,0 \mathrm{~mm}\end{array}$ & $\begin{array}{l}<1,0 \\
\mathrm{~mm}\end{array}$ \\
\hline CAC (1) & $510 \mathrm{~b}$ & $26 \mathrm{~h}$ & $46 \mathrm{e}$ & $72 \mathrm{~g}$ & $21 \mathrm{~d}$ & $21 \mathrm{~g}$ & $58 \mathrm{c}$ \\
\hline CAC (2) & $330 \mathrm{e}$ & $32 \mathrm{~g}$ & $53 \mathrm{~cd}$ & 85 cde & $20 \mathrm{~d}$ & $28 \mathrm{f}$ & $51 \mathrm{de}$ \\
\hline CRP & $410 \mathrm{~d}$ & $24 \mathrm{~h}$ & 59 a & 83 e & $22 \mathrm{~d}$ & 33 e & $45 \mathrm{f}$ \\
\hline CRSU (1) & $450 \mathrm{c}$ & $34 \mathrm{fg}$ & 43 ef & $77 \mathrm{f}$ & $7 \mathrm{fgh}$ & $41 \mathrm{~d}$ & $52 \mathrm{~d}$ \\
\hline CRSU (2) & $690 \mathrm{a}$ & $27 \mathrm{~h}$ & $51 \mathrm{~d}$ & $78 \mathrm{f}$ & $3 \mathrm{i}$ & $16 \mathrm{~h}$ & $82 \mathrm{a}$ \\
\hline CCPF & $160 \mathrm{ij}$ & $54 \mathrm{~b}$ & $35 \mathrm{~g}$ & $89 \mathrm{abc}$ & $14 \mathrm{e}$ & $48 \mathrm{c}$ & $38 \mathrm{~g}$ \\
\hline CCPG & $150 \mathrm{ij}$ & $64 \mathrm{a}$ & $27 \mathrm{~h}$ & $91 \mathrm{ab}$ & $65 a$ & $16 \mathrm{~h}$ & $20 \mathrm{~h}$ \\
\hline ССРМ (1) & $250 \mathrm{fg}$ & $40 \mathrm{de}$ & $46 \mathrm{e}$ & 86 cde & $37 \mathrm{~b}$ & $56 \mathrm{~b}$ & $7 \mathrm{i}$ \\
\hline CСРM (2) & $170 \mathrm{hi}$ & $48 \mathrm{c}$ & $39 \mathrm{f}$ & 87 abcd & 4 hi & $55 \mathrm{~b}$ & $40 \mathrm{~g}$ \\
\hline CPM & 190 hi & $31 \mathrm{~g}$ & $58 \mathrm{ab}$ & $89 \mathrm{abc}$ & $7 \mathrm{fg}$ & $41 \mathrm{~d}$ & $52 \mathrm{~d}$ \\
\hline CPC & $170 \mathrm{hi}$ & $41 \mathrm{~d}$ & $51 \mathrm{~d}$ & $92 \mathrm{a}$ & $22 \mathrm{~d}$ & $30 \mathrm{f}$ & $48 \mathrm{e}$ \\
\hline CPF & $210 \mathrm{gh}$ & 37 ef & $55 \mathrm{bc}$ & $92 \mathrm{a}$ & $9 \mathrm{f}$ & $33 \mathrm{e}$ & $58 \mathrm{c}$ \\
\hline $\mathrm{CP}$ & $270 \mathrm{f}$ & $24 \mathrm{~h}$ & $60 \mathrm{a}$ & $84 \mathrm{de}$ & $6 \mathrm{gh}$ & $42 \mathrm{~d}$ & $53 d$ \\
\hline COUA & $250 \mathrm{f}$ & $44 \mathrm{~d}$ & $41 \mathrm{f}$ & 85 cde & $25 c$ & $58 \mathrm{a}$ & $17 \mathrm{~h}$ \\
\hline СВCA & $120 \mathrm{ij}$ & $52 \mathrm{~b}$ & $41 \mathrm{f}$ & $92 \mathrm{a}$ & $3 \mathrm{i}$ & $33 \mathrm{e}$ & $64 \mathrm{~b}$ \\
\hline
\end{tabular}

* Compost de ave de corral (CAC (1) y (2)), compost de residuos porcino (CRP), compost de residuos sólidos urbanos (CRSU (1) y (2)), compost corteza de pino fina (CCPF), compost corteza de pino gruesa (CCPG), compost corteza de pino media (CCPM (1) y (2)), compost de poda de mora (CPM), compost de poda de cedro (CPC), compost de poda de fresno (CPF), compost de poda (CP), compost de orujo de uva agotado (COUA) y compost de bagazo de caña azúcar (CBCA). ). ${ }^{* *}$ Letras distintas entre filas de una misma columna indican diferencias significativas $(\mathrm{p} \leq 0,05)$ para el test de Tukey.

* Poultry compost (CAC (1) and (2)), pig waste compost (CRP), urban solid waste compost (CRSU (1) and (2)), fine pine bark compost (CCPF), thick pine bark compost (CCPG), medium pine bark compost (CCPM (1) and (2)), mulberry prune compost $(\mathrm{CPM})$, cedar pruning compost $(\mathrm{CPC})$, ash pruning compost (CPF), pruning compost $(\mathrm{CP})$, grape marc compost (COUA) and sugarcane bagasse compost (CBCA). ${ }^{* *}$ Different letters between rows of the same column indicate significant differences $(\mathrm{p} \leq 0.05)$ for the Tukey test.

apropiado entre 55 y 70\% (Abad et al., 2001). El CCPG y el CCPF tuvieron los menores valores de capacidad de retención de agua.

Pero si bien estos rangos de referencia ayudan a conocer o caracterizar las propiedades de un componente o sustrato, no son tan estrictos y deberían ser ajustados de acuerdo a la necesidad de aireación o requerimiento hídrico para cada especie a cultivar, el sistema de riego, el tamaño del recipiente empleado, entre otros factores. Por ejemplo, un sustrato apropiado para un techo verde extensivo debería tener una porosidad de aireación elevada (superior al rango) debido a las especies de bajos requerimientos hídricos que se utilizan (suculentas) (Farrell et al. 2012). En adición, en ensayos realizados por Owen y Altland (2008) observaron que el aumento de la altura del recipiente resultó en una disminución lineal en la capacidad de retención de agua y un aumento lineal en la porosidad de aireación. Es decir que a menor altura del recipiente se requieren sustratos más aireados.

En cuanto al tamaño de partículas (Tabla 4), el compost que tuvo mayor porcentaje de partículas $>3,35 \mathrm{~mm}$ fue el CCPG, seguido por el CCPM (1) diferenciándose entre ambos y con los restantes compost $(\mathrm{P}<0,05)$. El CCPM (2), el CBCA y el CRSU (2) fueron los de menor porcentaje sin diferencia entre los mismos. El mayor porcentaje de partículas entre 3,35 a $1 \mathrm{~mm}$ fue en el COUA diferenciándose de todos los compost $(\mathrm{P}<$ 0,05), seguido por los CCPM (1) y (2). El menor porcentaje fue en el CRSU (2) y CCPG sin diferencias entre ambos. Finalmente, el mayor 
porcentaje de partículas $<1 \mathrm{~mm}$ fue en el CRSU (2), seguido por el CBCA, ambos con diferencias significativas entre sí y con los demás compost. El CCPM (1) fue el de menor porcentaje y se diferenció de los otros compost. El porcentaje de tamaño de partículas es una de las propiedades físicas con mayor influencia sobre las demás propiedades, especialmente en la porosidad (Raviv y Lieth, 2008). Las partículas mayores a $1 \mathrm{~mm}$ dan lugar a poros grandes aportando aireación y las menores a $1 \mathrm{~mm}$ conforman poros medianos a pequeños proporcionando retención de agua. Por coincidencia, aquellos compost con una alta porosidad de aireación fueron los que tuvieron mayor porcentaje de partículas superiores a $1 \mathrm{~mm}$, destacándose los compost de corteza de pino y orujo de uva agotado.

\section{CONCLUSIONES}

En conclusión, todos los compost son viables para su uso, siempre y cuando sean utilizados en un porcentaje en la mezcla acorde a las propiedades que posee, y de las características que debe tener el sustrato según el tamaño/ altura del recipiente y condiciones del cultivo. Desde el punto de vista físico, los compost de corteza de pino podrían ser componentes que aportarían aireación pudiéndose mezclar con otros materiales de alta capacidad de retención de agua. Si son empleados en forma pura habría que tener en cuenta que el riego debería ser más frecuente. La mayor atención a la hora de formular un sustrato con los compost evaluados sería con respecto a las propiedades químicas, la mayoría de estos compost superan el rango adecuado de $\mathrm{pH}(5,5-6,3)$, excepto algunos como los elaborados con corteza de pino. Si bien gran parte de los compost no tuvieron una CE elevada, hubo algunos como el CAC, el CRSU y el CRP que superaron $1 \mathrm{dS} \mathrm{m}^{-1}$, y podrían causar efectos nocivos en las plantas si se incorpora en altas proporciones en la formulación del sustrato. La CE se correlacionó en especial con las altas concentraciones de potasio y sodio por lo cual se debe tener en cuenta para programar el plan de fertilización.

Se destaca la importancia de realizar un análisis físico y químico previo al compost que se empleará para formular un sustrato, y así poder decidir con criterio el porcentaje adecuado que se incorporará a la mezcla. Para próximas investigaciones se sugiere realizar análisis de metales pesados, principalmente en los casos en que el sustrato será empleado para producción de productos alimenticios como hortalizas.

\section{LITERATURA CITADA}

Abad, M., P.F. Martínez, M.D Martínez, y J. Martínez. 1993. Evaluación agronómica de los sustratos de cultivo. Actas de Hort. 11:141-154.

Abad, M., P. Noguera, and S. Burés. 2001. National inventory of organic wastes for use as growing media for ornamental potted plant production: case study in Spain. Bioresource Technology 77(2):197-200.

Abad, M., P. Noguera, y C. Carrion. 2004. Los sustratos en los cultivos sin suelo. p. 113-158. En Urrestarazu Gavilan M. (ed.) Tratado de cultivo sin suelo. $3^{\underline{a}}$ ed. Mundi Prensa, Madrid, España.

Abbas, M., M. Aftab, M. U.H. Zafar, Q. Iqbal, M. Hussain, and M.M. Khan. 2015. Effect of organically amended growing substrates on the growth and physiological attributes of citrus plants. Communications in Soil Science and Plant Analysis 46(15):1863-1880.

Ansorena, J. 1994. Sustratos propiedades y caracterización. p. 172. 1 $1^{\underline{a}}$ ed. Mundi Prensa, Madrid, España.

Aguilera, R.M., A. Aldrete, T.T. Martínez, y V.M.C. Ordá. 2016. Producción de Pinus montezumae Lamb. con diferentes sustratos y fertilizantes de liberación controlada. Agrociencia 50(1):107-118.

Barbaro, L.A., M.A. Karlanian, y D.E. Morisigue. 2010. Utilización de azufre micronizado en la corrección del pH de compost de residuos de poda. Agriscientia 27(2):125-130.

Barbaro, L.A., M.A. Karlanian, S. Imhoff, y D.E. Morisigue. 2011. Caracterización de la turba subtropical del departamento Islas del Ibicuy (Entre Ríos, Argentina). Agriscientia 28(2):137-145.

Barbaro, L.A., M.A. Karlanian, P.F. Rizzo, N.I. Riera, V. Della Torre, M. Beltrán, et al. 2013. Compost de guano de gallina en la composición de sustratos para la producción de plantines florales. Agriscientia 30(1):25-35.

Barbaro, L.A., A. Mazzoni, M.A. Karlanian, M.N. Fernandez, y D.E. Morisigue. 2014. Cenizas del volcán Puyehue como sustrato para plantas. Horticultura Argentina 33(81):44-53.

Barbaro, L.A., M.A. Karlanian, y D.A. Mata. 2018. Importancia del $\mathrm{pH}$ y la Conductividad Eléctrica (CE) en los sustratos para plantas. p. 10. Ediciones INTA, Buenos Aires, Argentina.

Barrett, G.E., P.D. Alexander, J.S. Robinson, and N.C. Bragg. 2016. Achieving environmentally sustainable growing media for soilless plant cultivation systems. A review. Scientia Horticulturae 212:220-234. 
Biaggi, C., F. Mondzak y A. Valeiro. 2011. Problemas ambientales, oportunidades de desarrollo: la experiencia del Proyecto INTA Prozono. 117 p. Ediciones INTA, Tucumán, Argentina.

Bunt, B.R. 2012. Media and mixes for containergrown plants: a manual on the preparation and use of growing media for pot plants. 309 p. $2^{\mathrm{a}}$ ed. Springer Science \& Business Media, Londres, Inglaterra.

Bures, S. 1997. Sustratos. 342 p. $1^{\text {a }}$ ed. Agrotecnias, Madrid, España.

Cáceres, R., N. Coromina, K. Malińska, and O. Marfà. 2015. Evolution of process control parameters during extended co-composting of green waste and solid fraction of cattle slurry to obtain growing media. Bioresource Technology 179:398-406.

Carlile, W.R., C. Cattivello, y P. Zaccheo. 2015. Organic growing media: Constituents and properties. Vadose Zone Journal 14(6). p. 0125.

Carmona C.E., y B.M. Abad. 2008. Aplicación del compost en viveros y semilleros. p. 399-424. En Moreno Casco, J., y R. Moral Herrero (eds.) Compostaje. Mundi Prensa, Madrid, España.

Carrión, C., M. Abad, F. Fornes, V. Noguera, R. Puchades, A. Maquieira, et al. 2005. Acidification of composts from agricultural wastes to prepare nursery potting mixtures. Acta Hortic. 779:333-340. doi:10.17660/ ActaHortic.2008.779.41

Chong, C., R.A. Cline, and D.L. Rinker. 1991. Growth and mineral nutrition status of containerized woody species in media amended with spent mushroom compost. J. Am. Soc. Horticult. Sci. 116:242-247.

Cruz, C.E., C.A. Can, V.M. Sandoval, M.R. Bugarin, B.A. Robles, y L.P. Juarez 2013. Sustratos en la horticultura. Revista Bio Ciencias 2(2):17-26.

De Boodt, M., O. Verdonck, and J. Cappaert. 1974. Methods for measuring the water release curve of organic substrates. Acta Hortic. 37:2054-2062. doi: 10.17660/ ActaHortic.1974.37.20

Di Rienzo, J.A., F. Casanoves, M.G. Balzarini, L. Gonzalez, M. Tablada, y C.W. Robledo. 2009. InfoStat versión 2009. Grupo InfoStat, FCA, Universidad Nacional de Córdoba, Argentina. Disponible http://www.infostat. com.ar. página principal (Consulta 10 mayo 2018)
Farrell, C., R.E. Mitchell, C. Szota, J.P. Rayner, and N.S.G. Williams. 2012. Green roofs for hot and dry climates: interacting effects of plant water use, succulence and substrate. Ecological Engineering 49:270-276.

Fermino, M.H. 2003. Métodos de análisis para caracterización física de sustratos para plantas. 89 p. Tesis doctoral, Universidad Federal de Rio Grande Do Sul. Puerto Alegre, Brasil.

Garcia G.A., M.P. Bernal, and A. Roig. 2002. Growth of ornamental plants in two composts prepared from agroindustrial wastes. Bioresource Technology 83(2):81-87.

Gayosso-Rodríguez, S., L. Borges-Gómez, E. Villanueva-Couoh, M.A. Estrada-Botello, y R. Garruña Hernández. 2016. Sustratos para producción de flores. Agrociencia 50(5):617631.

Gonzaga, L.D.M., S.S.D. Silva, S.D.A. Campos, R.D.P. Ferreira, A.N.D.R. Campos, and A.C.M.C.M. Cunha. 2016. Evaluation of substrates and amf sporulation in the production of seedlings of native forest species. Revista Árvore 40(2):245-254.

Guerrero, F., J.M. Gascó, and L. A. Hernández. 2002. Use of pine bark and sewage sludge compost as components of substrates for Pinus pinea and Cupressus arizonica production. J. Plant Nutr. 25(1):129-141.

Kleiber, T., B. Markiewicz, and A. Niewiadomska. 2012. Organic substrates for intensive horticultural cultures: Yield and nutrient status of plants, Microbiological Parameters of Substrates. Pol. J. Environ. Stud. 21(5):1261-1271

Krieger, S., C. Herrando, S. Gómez, y R. Cardozo. 2010. Evaluación de compost y lombricompost como sustratos para plantines de pimiento para pimentón (Capsicum annum L.) Ciencia 13(5):25-33.

Landis, T.D., R.W. Tinus, S.E. Mcdonald, y J.P. Barnett. 2000. Manual de viveros para producción de especies forestales en contenedor. p. 1-67. Manual agrícola. Departamento de Agricultura de los Estados Unidos, Servicio Forestal. Washington, Estados Unidos

Landis, T.D., and N. Morgan. 2009. Growing media alternatives for forest and native plant nurseries. p. 26-31. In Dumroese, R.K.; Riley, L.E. (eds.) National Proceedings: Forest and Conservation Nursery Associations-2008. Proc. RMRS-P-58. U.S. Department of Agriculture, Forest Service, Rocky Mountain Research Station, Fort Collins, Colorado, USA. 
Morales-Maldonado, E.R., y F. CasanovaLugo. 2015. Mezclas de sustratos orgánicos e inorgánicos, tamaño de partícula y proporción. Agronomía Mesoamericana 26:365-372.

Normann, K.A., R.T. Jun, y P.T. Vital De Siqueira. 2006. Floricultura, técnicas de preparo de substratos. Tecnologia fácil, 19. Ed. LK., Brasilia, Brazil.

Owen, J.S., and J.E. Altland. 2008. Container height and douglas fir bark texture affect substrate physical properties. HortScience 43(2):505-508.

Palencia, P., J.G. Bordonaba, F. Martínez, and L.A. Terry. 2016. Investigating the effect of different soilless substrates on strawberry productivity and fruit composition. Scientia Horticulturae 203:12-19.

Putra, P.A., and H. Yuliando. 2015. Soilless culture system to support water use efficiency and product quality: a review. Agriculture and Agricultural Science Procedia 3:283-288.
Raviv, M., and Lieth J.H. 2008. Soilless culture: theory and practice. 587 p. $1^{\text {a }}$ ed. Elsevier, London, England.

Sallesses, L.F., P.F. Rizzo, N. Riera, V. Della Torre, D.E. Crespo, y P.S. Pathauer. 2015. Efecto de compost de guano avícola en la producción de clones híbridos de Eucaliptus granáis $x$ Eucalyptus camaldulensis. Ciencia del Suelo 33(2):221-228.

Sonneveld, C. and Voogt, W. 2009. Substrates: Chemical characteri stics and preparation. $p$. 227-256. In Sonneveld, C. and Voogt, W. Plant nutrition in future greenhouse production. Springer, Heidelberg, Netherlands.

Urrestarazu, M. 2015. Manual Práctico del Cultivo sin Suelo e Hidroponía. 278 p. Mundi-Prensa. Madrid, España.

Vence, L.B. 2008. Disponibilidad de agua-aire en sustratos para plantas. Ciencia del Suelo 26(2):105-114. 